\title{
Construction and Quality Evaluation of the Japanese Rehabilitation Nutrition Database
}

\author{
Miyuki TAKASAKI ${ }^{1}$, Ryo MOMOSAKI ${ }^{2}$, Hidetaka WAKABAYASHI ${ }^{3}$ and Shinta NISHIOKA ${ }^{4}$ \\ ${ }^{1}$ Division of Nutrition Support, Tsurumakionsen Hospital, Hadano, Kanagawa 257-0001, Japan \\ ${ }^{2}$ Department of Rehabilitation Medicine, Teikyo University School of Medicine University Hospital, \\ Mizonokuchi, Kanagawa 213-8507, Japan \\ ${ }^{3}$ Department of Rehabilitation Medicine, Yokohama City University Medical Center, \\ Yokohama, Kanagawa 232-0024, Japan \\ ${ }^{4}$ Department of Clinical Nutrition and Food Services, Nagasaki Rehabilitation Hospital, \\ Nagasaki, Nagasaki 850-0854, Japan
}

(Received November 13, 2017)

\begin{abstract}
Summary Nutritional complications frequently occur among patients undergoing rehabilitation, and the importance of nutrition in these patients has been emphasized. However, there is not enough evidence available on rehabilitation nutrition. The Japan Rehabilitation Nutrition Database was set up to reflect the real-world clinical practice of rehabilitation nutrition. This paper describes the construction and quality evaluation of the registry database. We constructed a large-scale database that can be used for the clinical research of nutrition for rehabilitation. To verify the data, a simple comparison of the numbers of cases, data loss, data duplication, data type errors, out of range values, and input errors in the option columns was performed. From March 2016 to June 2017, 797 cases were registered in the rehabilitation database from 18 facilities. The variable entry error frequency ranges from $0 \%$ to $0.4 \%$ and the frequency of a main item missing from $0.4 \%$ to $10.9 \%$. Energy intake on hospitalization was missing in $10.9 \%$ of cases, and Mini Nutritional Assessment Short Form and Food Intake LEVEL Scale on hospitalization was missing in 9.7\% of cases. Stroke accounted for $45.7 \%$ of the diseases registered, pneumonia for $36.5 \%$, and proximal femoral fracture for $17.8 \%$. Through the use of this database, research on rehabilitation nutrition can be conducted, and there is a possibility that useful results for future clinical practice may be obtained. Verification of the secondary use of the database is becoming the basis for evidence-based nutritional rehabilitation.
\end{abstract}

Key Words Japan Rehabilitation Nutrition Database, Research Electronic Data Capture (REDCap), Electronic Data Capture (EDC), real-world clinical practice

Nutritional complications frequently occur among patients undergoing rehabilitation. In Japan, the concept of rehabilitation nutrition was proposed, and the Japanese Association of Rehabilitation Nutrition was subsequently launched. Rehabilitation nutrition for people with a disability and frail elderly people is defined as 1) holistic evaluation by the "International Classification of Functioning, Disability and Health" of the presence and causes of nutritional disorders, sarcopenia, and excessive or deficient intake of nutrients, 2) rehabilitation nutrition diagnosis and rehabilitation and nutrition goal-setting, and 3) achieving the highest level of physical functioning, activities, participation, and quality of life by improving nutritional status, sarcopenia, and frailty using "nutritional care management that takes into account rehabilitation" and "rehabilitation that considers nutrition" (1).

However, there is not enough evidence available on rehabilitation nutrition. Clinical studies that use a registry database offer one means of performing obser-

E-mail: takasaki-m@tsurumaki-hp.jp vational research. When reaching a conclusion in an observational study, it is important to adjust for various variables, which requires more than a certain number of cases. If multiple institutions jointly register their data about a specific disease in the same format, it is then possible to gather the relevant information easily in a relatively short time. This is a feasible and excellent methodology for research. However, reliable and comprehensive clinical data on nutritional status, nutritional management, and rehabilitation patients are limited. To strengthen the evidence regarding current rehabilitation nutrition, we have constructed a database, namely, the Japan Rehabilitation Nutrition Database, that can be utilized by multiple centers.

Objectives of the database. The overall objectives of the Japanese Rehabilitation Nutrition Database are as follows: 1) to publish clinical research on rehabilitation nutrition that will contribute to improving the quality of rehabilitation medicine around the world (i.e., a caseregistered database to enable observational research on rehabilitation nutrition), 2) to gather evidence that can contribute to policy recommendations relating to reha- 
Table 1. Patient input items on hospitalization.

\begin{tabular}{|c|c|}
\hline Basic information & Age, sex, onset (injury) day, hospitalization (change room), rehabilitation start date \\
\hline Diagnosis & $\begin{array}{l}\text { Aspiration pneumonia, non-aspiration pneumonia, cerebral infarction, cerebral hemorrhage, } \\
\text { subarachnoid hemorrhage, femoral neck fracture, femoral trochanteric fracture }\end{array}$ \\
\hline Surgical information & $\begin{array}{l}\text { Enter only stroke or fracture cases } \\
\text { Neurosurgical surgery (craniotomy, other, none), orthopedic surgery (artificial bone head } \\
\text { replacement surgery, osteosynthesis, other, none), neurosurgical surgery (craniotomy, other, } \\
\text { none), orthopedic surgery (artificial bone head replacement surgery, osteosynthesis, other, } \\
\text { none) }\end{array}$ \\
\hline Neurosurgical operation & Craniotomy, other, none \\
\hline Orthopedic surgery & Artificial bone head replacement surgery, osteosynthesis, others, none \\
\hline Pneumonia severity & $\begin{array}{l}\text { Only pneumonia cases input: A-DROP }{ }^{1} \text { 1) Male aged } 70 \text { y or over, female } 75 \text { y old or over; } \\
\text { 2) Blood urea nitrogen } 21 \mathrm{mg} / \mathrm{dL} \text { or more, or dehydrated; } 3) \mathrm{SpO}_{2} 90 \% \text { or less }\left(\mathrm{PaO}_{2} 60 \text { torr or }\right. \\
\text { less); 4) consciousness disorder; 5) systolic blood pressure } 90 \mathrm{mmHg}\end{array}$ \\
\hline Comorbidity & $\begin{array}{l}\text { Charlson comorbidity index sub-item (myocardial infarction, congestive heart failure, periph- } \\
\text { eral vascular disease, cerebrovascular disorder, dementia, chronic lung disease, collagen } \\
\text { disease, digestive ulcer, diabetic liver disease, diabetes without complications, hemiplegia } \\
\text { secondary diabetes with complications, diabetes with complications, solid cancer, leukemia, } \\
\text { lymphoma, moderate to high degree of liver function disorder), bone and joint disease, other } \\
\text { neurological disease, presence or absence of pretreatment tracheostomy, prenatal gastrostomy } \\
\text { nutrition, presence or absence }\end{array}$ \\
\hline
\end{tabular}

Before onset (pre-injury) presence or absence of nursing-care requirement, presence or
absence of co-dwellers, pre-hospital home environment (facility, hospital, home, other)

\begin{tabular}{ll}
\hline Drugs used & Presence of antipsychotics, anxiolytics, antidepressants \\
\hline In-patient test value & Albumin (g/dL), pneumonia only C-reactive protein (mg/dL) \\
\hline Rehabilitation information & $\begin{array}{l}\text { In cases of stroke and fracture, FIM }{ }^{2} \text { (all lower items) on hospitalization, Barthel index (all } \\
\text { lower items) on hospitalization, Food Intake LEVEL Scale on hospitalization, JCS } \\
\text { digits) on hospitalization, only stroke cases hospitalized Time mRS (modified Rankin Scale) }\end{array}$ \\
\hline $\begin{array}{l}\text { Nutrition-related } \\
\text { information }\end{array}$ & $\begin{array}{l}\text { Height (cm), weight (kg), MNA-SF }{ }^{4} \text { on hospitalization (also with lower item points), energy } \\
\text { intake (average for 1 wk after hospitalization) (kcal/d) }\end{array}$ \\
\hline $\begin{array}{l}\text { Nutrition form on } \\
\text { hospitalization } \\
\text { Route of administration }\end{array}$ & $\begin{array}{l}\text { Oral intake: 1) No oral intake; } 2) \text { jelly, mixture meal, semi-solid meals (foods that barely } \\
\text { require chewing); 3) soft food, soft vegetable diet (foods requiring chewing); } 4 \text { ) ordinary meals }\end{array}$ \\
\hline $\begin{array}{l}\text { Drinking water } \\
\text { Nil per os }\end{array}$ & $\begin{array}{l}\text { Presence or absence of peripheral vein nutrition, presence or absence of central venous nutri- } \\
\text { tion, presence or absence of tube feeding (gastric wax nutrition, nasal nutrition, etc.), pres- } \\
\text { ence or absence of use of semi-solid nutritional materials }\end{array}$ \\
\hline
\end{tabular}

\footnotetext{
${ }^{1}$ A-DROP: Age, Dehydration, Respiration, Orientation, Pressure.

${ }^{2}$ FIM: Functional Independence Measure.

${ }^{3}$ JCS: Japan Coma Scale.

${ }^{4}$ MNA-SF: Mini Nutritional Assessment Short Form.
}

bilitation nutrition, and 3) to provide research opportunities to members of the Rehabilitation Nutrition Society.

The specific objectives of this database project are as follows: 1) to collect data from each facility and build a case registration database on rehabilitation nutrition,
2) to provide information on the collected data according to the needs of the participating facilities, and 3) to allow each facility to utilize the data for clinical research purposes. Currently, we collect data on pneumonia in older patients from general wards and on strokes and hip fractures in patients from rehabilitation wards. 
Table 2. Clinical endpoints.

Hospital discharge date, General ward, recovery period ward, medical treatment-type ward, home, facility, death
discharge details (including transfers)

\begin{tabular}{ll}
\hline Dental-related & Presence or absence of dental intervention, presence of dental hygienist intervention \\
\hline Nutrition-related & $\mathrm{MNA}^{-S^{5}}$ at the time of discharge (also sub-items), body weight at discharge \\
\hline
\end{tabular}

${ }^{1} \mathrm{PT}$ : physical therapist.

${ }^{2}$ OT: occupational therapist.

${ }^{3}$ ST: speech-language-hearing therapist.

${ }^{4}$ FIM: Functional Independence Measure.

${ }^{5}$ MNA-SF: Mini Nutritional Assessment Short Form.

To advance clinical research on Japanese rehabilitation nutrition, our aim is to construct a Japanese rehabilitation nutrition database and evaluate its quality.

\section{MATERIALS AND METHODS}

Overview of the system. The Rehabilitation Nutrition Database is a web-based registry database. Attempts to construct a database that requires special variables inevitably leads to missing data and degrades the quality of the data collected. Electronic data capture (EDC), a system for the electronic collection of clinical research data, is an indispensable tool that enables high-quality data management. The merit of EDC includes the easy cleaning of data and real-time monitoring of data. Many EDC systems are expensive, but in recent years, cost-effective and easy-to-use EDCs have been developed. Research Electronic Data Capture (REDCap) is listed as a world standard medical research EDC (2). This EDC was developed by Vanderbilt University and its source code is provided free of charge. Currently, more than 130,000 REDCap projects are in progress throughout the world. Using REDCap enables databases to be built and easily managed at low cost on the web even if users are not experts in data management. User support for REDCap has recently been launched in Japan and applications for REDCap as an EDC for registry databases have been made by, for example, the Japan Rehabilitation Nutrition Research Group. Database users can log into the system from anywhere with a user name and password.

Database design. The Rehabilitation Nutrition Database is an Active Server web page constructed using REDCap. The input items can be confirmed as a list on the screen for case registration. The operation and man- agement of the database is carried out by the Japan Rehabilitation Nutrition Database Committee. The collected data are available for research purposes.

Inclusion and exclusion criteria. Participating facilities include general hospital wards and rehabilitation wards to which the members of the Japanese Association of Rehabilitation Nutrition belong. The recruitment of a facility requires participation in research by members of the Japanese Association of Rehabilitation Nutrition. Once the consent to participate in research is acquired, facilities that meet the criteria are registered (volunteer sampling) (Supplemental Online Materials, Table S1). The target patients are those who are admitted for rehabilitation purposes who have convalescence disorders, cerebral infarction, cerebral hemorrhage, subarachnoid hemorrhage, femoral neck fracture, femoral trochanter fracture (patients over $20 \mathrm{y}$ old), or for the treatment of pneumonia in elderly people ( $65 \mathrm{y}$ old and over) who are admitted to hospital. From March 2016, all target cases have been registered on admission to hospital and followed up until discharge. Database staff at each facility monitor the eligible cases. Since all data submitted to REDCap are non-traceable and anonymous, there is no need to obtain permission or explain the system to individual registered patients.

Data collection (variable input manual). The database has three main data input pages (basic information, input items during hospitalization, and input items at hospital discharge), and information on each facility is gathered and registered as an appendix when registering for a database project. Patient input items on hospitalization are shown in Table 1, and input items at discharge, that is clinical end points, are shown in Table 2. 
Table 3. Variable input error.

\begin{tabular}{lccl}
\hline & \multicolumn{2}{c}{ Number } \\
\cline { 2 - 3 } & Effective & Missing (\%) \\
\hline Data type error & 797 & $0(0.00)$ & $\begin{array}{l}\text { e.g., A nominal scale entered in the continuous scale input } \\
\text { column }\end{array}$ \\
\hline Outside range value & 797 & $1(0.13)$ & $\begin{array}{l}\text { Numerical values outside the permissible range are entered } \\
\text { Error content was 0 kg when discharged. }\end{array}$ \\
\hline Input error in the options column & 797 & $0(0.00)$ & e.g., Multiple selections in the selection field \\
\hline Contradictions between data & 797 & $3(0.38)$ & Three discharge (transfer) dates before hospitalization \\
\hline
\end{tabular}

Table 4. Missing major items.

\begin{tabular}{lrr}
\hline & & Number \\
\cline { 2 - 3 } & Effective & Missing (\%) \\
\hline Age & 791 & $6(0.75)$ \\
Sex & 791 & $6(0.75)$ \\
Major disease classification & 794 & $3(0.38)$ \\
Height on admission & 753 & $44(5.52)$ \\
Weight on admission & 756 & $41(5.14)$ \\
Onset (injury) & 722 & $75(9.41)$ \\
Hospitalization (change) day & 723 & $74(9.28)$ \\
Food Intake LEVEL Scale (FILS) (10) on admission & 720 & $77(9.66)$ \\
Mini Nutritional Assessment Short Form (MNA-SF) score on admission & 720 & $77(9.66)$ \\
Initial hospital energy intake & 710 & $87(10.92)$ \\
Number of hospital days & $713(-)$ & - \\
Weight at discharge & $713(-)$ & - \\
Number of rehabilitation units visited during hospitalization & $713(-)$ & - \\
MNA-SF scores at discharge & $713(-)$ & - \\
FILS at discharge & $713(-)$ & - \\
\hline
\end{tabular}

The criteria for the input methods of each item relating to the diagnosis, rehabilitation, nutrition, and medication are preset.

1) Diagnosis: Diagnoses of primary disease and comorbid disorders are based on the doctor's assessment. Refer to the ICD-10 code for details on the classification of stroke and hip fracture, cerebral infarction (I63), cerebral hemorrhage (I61), subarachnoid hemorrhage (I60), femoral neck fracture (S720), and femoral trochanter fracture (S721) (3).

A diagnosis of pneumonia requires the presence of an invasive shadow on chest X-ray or computed tomography, and at least two symptoms from among fever, high C-reactive protein value, raised white blood cell count, and airway symptoms.

A diagnosis of aspiration pneumonia is given according to the Japanese Respiratory Society Guidelines for the Management of Hospital-Acquired Pneumonia in Adults. The guidelines define aspiration pneumonia as pneumonia that develops in patients who have (or are strongly suspected to have) known dysphagia and aspiration. It is important to conduct swallowing function tests as a means of detecting dysphagia and aspiration and to determine whether aspiration has occurred during or after meals (4).

A definition of "fall" according to the FICSIT trials is "unintentionally coming to rest on the ground, floor, or other lower level" (5).

2) Comorbidity: Definitions of comorbidities included in the Charlson comorbidity index conform to ICD-10 classification (6). In addition, bone and joint diseases are classified as G10-G13, G20-G22, G254, G255, G312, G318, G319, G32, G35-G37, G40, G41, G931, G934, R470, and R56 (6). These diagnoses are based on the doctor's assessment.

3) Rehabilitation: The cost of rehabilitation is calculated by a physical therapist, occupational therapist. or speech-language-hearing therapist. Speech-languagehearing therapist intervention (including swallowing rehabilitation) is recommended according to the doctor's instructions.

4) Type of nutritional support: Information is gathered on nutritional administration route and nutrition type. Presence or absence of peripheral venous nutri- 
Table 5. Frequency distribution table.

\begin{tabular}{|c|c|c|c|c|c|}
\hline & \multicolumn{2}{|c|}{ Number } & \multirow{2}{*}{ Median } & \multicolumn{2}{|c|}{ Percentile } \\
\hline & Effective & Missing (\%) & & 25 & 75 \\
\hline Age (y) & 791 & $6(0.75)$ & 81.0 & 73.0 & 87.0 \\
\hline Height at hospitalization $(\mathrm{cm})$ & 753 & $44(5.52)$ & 155.0 & 148.0 & 163.0 \\
\hline Hospitalized weight $(\mathrm{kg})$ & 756 & $41(5.14)$ & 49.9 & 42.0 & 58.0 \\
\hline BMI $^{1}$ & 750 & $47(5.90)$ & 20.6 & 18.1 & 22.9 \\
\hline $\mathrm{CCI}^{2}$ total score & 797 & $0(0.00)$ & 1.0 & 0.0 & 2.0 \\
\hline Number of days from onset to hospitalization & 722 & $75(9.41)$ & 18.0 & 4.0 & 31.3 \\
\hline $\begin{array}{l}\text { Number of days between hospitalization and } \\
\text { rehabilitation }\end{array}$ & 617 & $180(22.58)$ & 0.0 & 0.0 & 0.0 \\
\hline $\mathrm{CRP}^{3}(\mathrm{mg} / \mathrm{dL})$ & 253 & $37(12.76)$ & 6.0 & 1.3 & 12.7 \\
\hline $\mathrm{Alb}^{4}(\mathrm{~g} / \mathrm{dL})$ & 693 & $104(13.05)$ & 3.5 & 3.1 & 3.9 \\
\hline Total FIM ${ }^{5}$ at admission & 503 & $1(0.20)$ & 69.0 & 40.0 & 91.0 \\
\hline Total $\mathrm{BI}^{6}$ on hospitalization & 218 & $72(24.83)$ & 20.0 & 0.0 & 55.0 \\
\hline MNA-SF ${ }^{7}$ total score on hospitalization & 720 & $77(9.66)$ & 6.0 & 4.0 & 8.0 \\
\hline Energy intake (kcal/d) & 710 & $87(10.92)$ & $1,400.0$ & $1,100.0$ & $1,516.3$ \\
\hline Hospital stay (d) & 695 & $102(12.80)$ & 62.0 & 29.0 & 100.0 \\
\hline Discharge weight (kg) & 670 & $127(15.93)$ & 48.9 & 41.0 & 57.0 \\
\hline PTOTST $^{8}$; total number & 403 & $71(14.98)$ & 816.0 & 594.0 & 1137.0 \\
\hline $\begin{array}{l}\text { Feeding function therapy (total number of inter- } \\
\text { ventions during hospitalization) }\end{array}$ & 796 & $1(0.13)$ & 29.0 & 5.3 & 70.5 \\
\hline Total FIM ${ }^{5}$ at discharge & 490 & $14(2.78)$ & 107.0 & 71.8 & 120.0 \\
\hline Total $\mathrm{BI}^{6}$ at discharge & 201 & $89(30.69)$ & 40.0 & 0.0 & 90.0 \\
\hline MNA-SF ${ }^{7}$ total score at discharge & 696 & $101(12.67)$ & 9.0 & 6.0 & 10.0 \\
\hline
\end{tabular}

${ }^{1}$ BMI: body mass index.

${ }^{2}$ CCI: Charlson Comorbidity Index.

${ }^{3}$ CRP: C-reactive protein.

${ }^{4}$ Alb: albumin.

${ }^{5}$ FIM: Functional Independence Measure.

${ }^{6}$ BI: Barthel index.

${ }^{7}$ MNA-SF: Mini Nutritional Assessment Short Form.

${ }^{8}$ PTOTST: physical therapist, occupational therapist, speech-language-hearing therapist.

tion, presence or absence of central venous nutrition, presence or absence of tube feeding (gastric wax nutrition, nasal nutrition, etc.), and presence or absence of use of semi-solid nutrient materials. Semisolid nutritional material, according to the Japanese Society for Functional Structure of Nutrition, is defined as "a nutrient directly injected into the stomach via a tube that increases viscosity or changes its shape from liquid" (7). In addition to the presence or absence of oral intake, in the case of presence, data on the type of meal and the thickening or not of drinking water are collected.

5) Medication: Information on the use of antipsychotics, anxiolytics, and antidepressants is collected at the time of admission. The classification of medicines follows "Today's Drug Therapy in 2015," Nankodo, 2015 (8).

6) Body weight at discharge: When weight at the time of discharge has not been measured, it can be entered only when the last measured weight value is within 1 mo before discharge and the measurement is performed separately from measurements taken on the day of hospitalization.

Ethical considerations. Each participating hospital must assign a person to be responsible for data trace- ability. Regarding personal information, only anonymous data that cannot be linked to patients are registered in the database. This study has been performed in accordance with the ethical standards established in the 1964 Declaration of Helsinki and later amendments. The database and this study was reviewed and approved by the Ethics Committee of The Jikei University School of Medicine (Authorization ID 27-150(8035)).

Data security and integrity. All data collection forms and data reports are given an automatically allocated REDCap ID. From the basic information, the patient's local identification number is registered, but the transmission of information to the center is managed via the REDCap identification number. Access to the system is managed via user name and password, and users need to periodically update their passwords. Only the Japan Rehabilitation Nutrition Database Committee administrator can add new users. Communication with the central server is by encrypted password-protected data via a secure server protocol. To ensure the accuracy of the data, registry guidelines, a variable input manual, and REDCap input manual are distributed by the Japan Rehabilitation Nutrition Database Committee to the data entry personnel at the participating facilities. After 
training, the input data are entered.

Statistical analysis. All analyses were performed using SPSS $^{\circledR}$ Statistics Version 24.0 for Microsoft Windows.

Variables with a skewed distribution are reported as the median and interquartile range. Variable input error was detected by the REDcap data quality evaluation report. Variable input errors are classified as shown in Table 3. Data including errors are excluded from statistical analysis. Missing values can be regarded as valid if the method of dealing with missing values is appropriate, especially if those methods are predefined during the protocol analysis plan (9). In this database, data including missing values are excluded from analysis targets.

\section{RESULTS}

\section{Current status}

Registration of the Rehabilitation Database began in March 2016. Currently 18 facilities are participating. As of the end of June 2017, the number of registered patients had reached 797. Variable input error (Table 3), missing major items (Table 4), and frequency distribution (Table 5) were examined from the input situation of the rehabilitation nutrition database this time.

Quality of data

1) Variable input error. As shown in Table 3, the variable input error was 0 for the data type error, 1 for the value out of the range, 0 for the input error of the option column, and for 3 inconsistencies between the data.

2) Missing major items. Of the 15 main items, 10 items were considered at the time of admission (Supplemental Online Materials, Table S2). Initial hospital energy intake had the most missing values (87 cases) while major disease classification had the fewest missing values (3 cases) (Table 4). Many cases (84) were not discharged as of the end of June (Supplemental Online Materials, Table S3), and missing main items were counted only on hospitalization (Table 4).

3) Descriptive statistics. The major diseases recorded were stroke $45.7 \%$ (363 cases), pneumonia $36.5 \%$ (290 cases), and proximal femoral fracture 17.8\% (141 cases). In the frequency distribution table, CRP and BI measured by pneumonia alone were calculated with 290 denominators. The frequency of main item missing was from $0.4 \%$ to $10.9 \%$. Energy intake on hospitalization was missing in $10.9 \%$ of cases, and the Mini Nutritional Assessment Short Form (MNA-SF) and the Food Intake LEVEL Scale (FILS) (10) on hospitalization were missing in $9.7 \%$ of cases. Initial hospital energy intake, for which there were many missing values, was $1,100 \mathrm{kcal}$ at the $25 \mathrm{th}$ percentile and $1,516 \mathrm{kcal}$ at $75 \mathrm{th}$ percentile. Rehabilitation was started in 501 (81.2\%) on the day of hospitalization because the registered hospitals had a system for early rehabilitation (Table 5). The quality of the data entered was reliable (Table 5).

\section{DISCUSSION}

We constructed a Japanese rehabilitation nutrition database and evaluated its quality. In creating this data- base, selection of data items to collect was important. We constructed the database and completed it by listening to the opinions of members of many professions.

The United States' Uniform Data System for Medical Rehabilitation (UDSMR) is the largest rehabilitation registry database in the world. In Japan, the Japanese Rehabilitation Database (JRD) specializes in rehabilitation medicine, and the number of English papers using the JRD is increasing (11-16). The Convalescent Rehabilitation Ward Association also maintains a database (17). Other important databases in the past have not incorporated data on both rehabilitation and nutrition. Unlike conventional rehabilitation databases, this database contains nutrition-specific items such as swallowing function, form of diet, nutritional intake, and many rehabilitation variables.

Although more than 200 evaluations on the quality of data have been proposed the definitions vary considerably from article to article (https://www.nihocollaboratory. org/Products/Assessing-dara-qualityV1\%200.pdf) (18). The variable entry error frequency ranges from $0 \%$ to $0.4 \%$ and the frequency of a main item missing from $0.4 \%$ to $10.9 \%$. By utilizing this database, research on rehabilitation nutrition can be conducted, and there is a possibility that the results could be made available for clinical practice in the future.

Limitations

There are diverse forms of food in Japan, and it is difficult to grasp the actual dietary content in swallowing disorders. However, data on meal route and the presence of a swallowing disorder, plus calorie intake on hospitalization have been collected, and the minimum information required for nutritional rehabilitation is available.

This study is insufficient for assessing the accuracy of the registered data and whether it can be used for research; thus it is necessary to validate the data through future research. However, research papers that made use of this database have already been accepted and it is becoming a basis for demonstrating the evidence on nutritional rehabilitation.

\section{Further research}

We wrote this paper on behalf of the Rehabilitation Database Group of the Research and Investigation Committee of the Japanese Rehabilitation Nutrition Society. The research objectives were to gain an understanding of the conditions in daily practice, to improve the quality of medical care, and prepare basic data for the revision of medical fees. Observational research, which is research based on observing the effects of a treatment being conducted and the outcome, is considered important. Although insufficient if obtained from only one hospital, research on actual clinical site raw data from multiple medical institutions is useful for establishing evidence on rehabilitation nutrition. The validity of the collected data items must be confirmed by secondary use of the database (19). Data entry personnel from participating facilities who have 10 or more registered cases in the database can receive support to help them with statistical analyses of secondary data and the preparation of theses. We would like to make this database more 
useful from the perspective of the multiple occupations registered among the Rehabilitation Database Group. By conducting high-quality observational research, realworld effectiveness can be verified.

\section{CONCLUSION}

We constructed a Japanese rehabilitation nutrition database and evaluated its quality.

Through the use of this database, research on rehabilitation nutrition can be conducted, and there is a possibility that useful results for future clinical practice may be obtained. Verification of the secondary use of the database is becoming the basis for evidence-based nutritional rehabilitation.

\section{Acknowledgments}

Construction of the Japan Rehabilitation Nutrition Database was supported by a Grant-in-Aid for Scientific Research from the Japan Society for the Promotion of Science (15K01395). Parts 1) and 2) of this project were supported by a scientific research grant to support and promote database research related to rehabilitation. The grant will be used for expenses related to the construction and maintenance of the database, for the input of data, and for the calibration costs pertaining to each researcher. The authors express appreciation to all participating facilities and registered personnel involved with the database.

Hospital name list: Atagawa Onsen Hospital, Kanazawa Nishi Hospital, Sakurakai Medical Corporation Sakurakai Hospital, Sapporonishimaruyama Hospital, Mihono Hospital, Tamana Regional Health Medical Center, Tsurumakionsen Hospital, Jikei University Daisan Hospital, Nagasaki Rehabilitation Hospital, Nakanoshima Iwaki Hospital, Nanko Hospital, Nishi Hiroshima Rehabilitation Hospital, Nishinomiya Kyoritsu Neurosurgical Hospital, Haradoi Hospital, Minamisoma Municipal General Hospital, Miharu Municipal Hospital,

\section{Supporting Information}

Supplemental Online Material is available on J-STAGE.

\section{REFERENCES}

1) Wakabayashi H. 2017. Rehabilitation nutrition in general and family medicine. J Gen Fam Med 18: 153-154.

2) Harris PA, Taylor R, Thielke R, Payne J, Gonzalez N, Conde JG. 2009. Research electronic data capture (RED ap) - a metadata-driven methodology and workflow process for providing translational research informatics support. J Biomed Inform 42: 377-381.

3) Kimberly OM, Karon FC, Matt DP, Kimberly RW, John FH, Carol MA. 2005. Measuring, diagnoses: ICD code accuracy. Health Services Res 40: 1620-1639.

4) Kohno S, Imanura Y, Shindo Y. 2013. Clinical practice guidelines for nursing- and healthcare-associated pneumonia (NHCAP). Respir Investig 51: 103-126.

5) Buchner DM, Hornbrook MC, Kutner NG, Tinetti ME, Ory MG, Mulrow CD, Schechtman KB, Gerety MB, Fiatarone MA, Wolf SL. 1993. Development of the common data base for the FICSIT trials. J Am Geriatr Soc 41: 297-308.

6) Quan H, Sundararajan V, Halfon P, Fong A, Burnand
B, Luthi JC, Saunders LD, Beck CA, Feasby TE. 2005. Coding algorithms for defining comorbidities in ICD9-CM and ICD-10 administrative data. Med Care 43: 1130-1139.

7) Goda F, Iijima S, Kanie J, Nishiguchi Y. 2009. Unification of nutrient formula and terminology. Clinical Nutrition 114: 645-650 (in Japanese).

8) Watanabe K, Yoshino A, Tanahasi N, Hayashida M. 2015. Drugs acting on the nervous system. In: Today's Drug Therapy in 2015 (Urabe A, Shimada K, Kawai S, eds), p 804-863. Nankodo, Tokyo (in Japanese).

9) International Conference on Harmonisation E9 Expert Working Group. 1999. ICH Harmonised Tripartite Guideline. Statistical principles for clinical trials. Stat Med 18: 1905-1942.

10) Kunieda K, Ohno T, Fujishima I, Hojo K, Morita T. 2013. Reliability and validity of a tool to measure the severity of dysphagia. The Food Intake LEVEL Scale. J Pain Symptom Manage 46: 201-206.

11) Hada T, Momosaki R, Abo M. 2018. Impact of orthotic therapy for improving activities of daily living in individuals with spinal cord injury: a retrospective cohort study. Spinal Cord. Advance Publication, doi: 10.1038/ s41393-018-0088-9.

12) Sawaguchi A, Momosaki R, Hasebe K, Chono M, Kasuga S, Abo M. 2018. Effectiveness of preoperative physical therapy for older patients with hip fracture. Geriatr Gerontol Int. Advance Publication, doi: 10.1111/ggi. 13290.

13) Kinoshita S, Momosaki R, Kakuda W, Okamoto T, Abo M. 2017. Association between 7 days per week rehabilitation and functional recovery of patients with acute stroke: a retrospective cohort study based on the Japan Rehabilitation Database. Arch Phys Med Rehabil 98: 701-706.

14) Momosaki R, Kakuda W, Yamada N, Abo M. 2015. Impact of board-certificated physiatrists on rehabilitation outcomes in elderly patients after hip fracture: An observational study using the Japan Rehabilitation Database. Geriatr Gerontol Int 16: 963-968.

15) Momosaki R, Abo M, Watanabe S, Kakuda W, Yamada N, Kinoshita S. 2015. Effects of ankle-foot orthoses on functional recovery after stroke: a propensity score analysis based on Japan Rehabilitation Database. PLoS One 10: $\mathrm{e} 0122688$.

16) Kinoshita S, Kakuda W, Momosaki R, Yamada N, Sugawara H, Watanabe S, Abo M. 2015. Clinical management provided by board-certificated physiatrists in early rehabilitation is a significant determinant of functional improvement in acute stroke patients: a retrospective analysis of Japan Rehabilitation Database. J Stroke Cerebrovasc Dis 24: 1019-1024.

17) Miyai I, Sonoda S, Nagai S, Takayama Y, Inoue Y, Kakehi A, Kurihara M, Ishikawa M. 2011. Results of new policies for inpatient rehabilitation coverage in Japan. Neurorehabil Neural Repair 25: 540-547.

18) Wang RY, Strong DM. 1996. Beyond accuracy: What data quality means to data consumers. J Management Information Systems 12: 5-33.

19) Nishioka S, Wakabayashi H, Momosaki R. 2018. Nutritional status changes and activities of daily living after hip fracture in convalescent rehabilitation wards: a retrospective observational cohort study from the Japan Rehabilitation Nutrition Database. J Acad Nutr Diet 118: 1270-1276. 